\title{
Distributed Energy Resources Network Connection Considering Reliability Optimization using a NSGA- II Algorithm
}

\author{
A. Vieira Pombo \\ Electrical Engineering Department \\ ESTSetúbal - Inst. Politécnico Setúbal \\ Setúbal, Portugal
}

\author{
J. Murta-Pina \\ CTS/UNINOVA \\ FCT - Universidade Nova de Lisboa \\ Caparica, Portugal
}

\author{
V. Fernão Pires \\ ESTSetúbal-Inst.Politécnico Setúbal \\ INESC-ID Lisboa \\ Setúbal, Portugal
}

\begin{abstract}
Distributed Energy Resources (DER) has been widely introduced in distribution networks in response to the increase of environmental awareness of the consumers. The benefits with the use of DER are increased with network reconfiguration, but in some countries exists the impossibility of island operation as well as the obligation to buy all the electricity generated. These limitations, in a network fault situation, causes a waste of resources, because of the unavailability of the DER. On other hand, the location of DER connection under the context of the improvement of the reliability indexes has not been studied. Thus, this paper will propose a multi objective optimization of the location of DER connection considering the switching devices placement to increase network reliability and availability of DER, minimizing at the same time the investment in equipment in a no island operation environment. For the resolution of the proposed formulation, it will be used the multi objective algorithm NSGA-II (Fast Non-dominated Sorting Genetic Algorithm). The formulation of the problem also considers a composite index made up of DER and interruption duration. This approach is applied to a real utility distribution network, with the results presented and discussed.
\end{abstract}

Keywords-Multiobjective Optimization; Switching Devices; NSGA-II Algorithm; Distributed Energy Resources; No-Island Operation.

\section{INTRODUCTION}

With the change in public opinion relatively to environmental issues, the importance of the generation of electricity by clean methods has become a critical issue. All over the world, we have been assisting to the change of electricity generation from conventional methods to the use of renewable sources like wind and solar, to reduce greenhouse gas emissions [1-4]. The impact of different Distribution Energy Resources (DER) in the electrical network is discussed in [5]. Relatively to traditional electrical generation, concentrated in large power plants, these renewable generations are characterized by its dissemination over the electrical grid. This places several new challenges to the electrical companies, and in particular to distribution companies. Besides the environmental issues, public opinion is also preoccupied with the quality of the electrical supply. So for the last years we have assisted to the imposition of regulation by regulatory bodies regarding quality standards. With these issues in mind, the optimal placement of switching devices has been used to improve reliability of distribution networks and DER availability. The optimal location of switching devices helps to reduce the number of consumers affected by an outage, thus improving network reliability, as well as improving DER availability.

The optimal switching device placement was used in [6] to minimize the cost of equipment, operation and maintenance and in [7] to minimize risk and operational cost. Switches and reclosers were in [8] both optimally located to enhance system reliability. Switches were optimally placed in [9] in a network with distributed generation to improve economic factors and reliability. Economic factors (investment, losses and customer outage cost) were also improved in [10]. In [11] network reliability with distributed generation was improved with the use of optimal recloser placement. In [12] reclosers were also used to improve equipment cost and energy not supplied. Protective devices and a risk analysis were used in [13] and [14] to improve network reliability. The optimal number and location of switching devices are determined in [15], [16] to improve network reliability and equipment cost.

Although DER availability is improved by optimal switching devices placement, it can be also improved if we optimally choose the connection point to the electrical distribution network. If we connect the DER to one point of the network with better reliability, we are also assuring better DER availability. An overview of the literature shows that the problem of distribution network reconfiguration and DER connection optimization has mainly been independently treated. A review is presented in [17] contemplating the stateof-the-art in multi objective DER planning, challenges, trends and latest developments. In [18] a state-of-the-art of models and methods in DER optimal allocation is presented. In [19] the allocation and size of distributed generation are optimized using a composite reliability index. DG placement is also optimized in [20] considering a trade between benefits and equipment cost. To improve losses and network reliability, distributed generation is optimally allocated in [21] and in [22] considering environmental constrains. 
When considering DER on distribution networks, normally it is considered island operation. But this kind of operation may not be allowed in some countries, due to the regulation of the operation of the electrical system. These regulations may in some countries establish contracts with DER operators that oblige the distribution system to buy all the electricity generated. In case of an outage, that implies the disconnection of the DER from the distribution network, there is a reduction of the availability of the DER and payments to the DER operators in spite of non-using the electricity generated. Thus, in [23] it was proposed a multiobjective optimization tacking into consideration the number and location of the switches to improve the network reliability and availability of the DER. However, this work was not focused in the location of the DER.

To increase distribution network reliability and to reduce the unavailability of the DER, this paper considers two strategies, 1) the optimal location of DER connection to the network and 2) the optimal location and number of switching devices. This strategy will be applied to a no island operation of the network. The optimal location and number of the switching devices in the network isolates parts of the network that are affected by an outage, thus reducing the consumers affected by an outage which in turn increases network reliability and DER availability. On the other hand, by optimally choosing the point of the DER connection we can increase its availability even more. The problem also considers the minimization of the equipment cost. This last objective function is in conflict with the other two objective functions. Thus, this problem will be treated by a multi objective optimization. This optimization will be resolved through the use of the multi objective algorithm NSGA-II (Fast Nondominated Sorting Genetic Algorithm). To test the proposed approach, a real distribution network was used.

\section{PROBLEM FORMULATION}

As referred, the increase of DER in distribution electrical networks has become a reality that has to be optimally explored in conjunction with other equipment's and regulations. On the other hand, quality issues also attracted the awareness of consumers witch led to the regulation and imposition of quality standards. In order to improve the reliability of the distribution networks, the optimal calculation of number and location of switching devices is a fundamental issue. However, with the integration of DER in radial distributed network this problem increases the complexity of the problem. In fact, considering no island operation, if the problem is only analysed under the point of view of the classical reliability indexes (such as SAIDI and SAIFI), the availability of the DER will be not considered. Due to this, there may be important financial and generated green energy losses. To overcome this problem, two important issues must be considered: 1) location of DER connection and 2) location of switching devices considering the location of DER. Many times the renewable sources must be located in a specific place. In this case, these sources are normally connected to the nearest point of the electrical distribution network. However, to improve the reliability of the network and the availability of the renewable sources several points of connection were considered. Since the cost of this connection is normally high, all of the network buses were not considered for possible connection points, but only nearest and possible points, as can be seen by Fig. 1. By this limitation there will be a limit on the increase of connection costs. The possibility to connect to another point of the network (even the nearest ones) allows an improvement in the availability of the renewable sources and reliability of the network under a limited increase of costs.

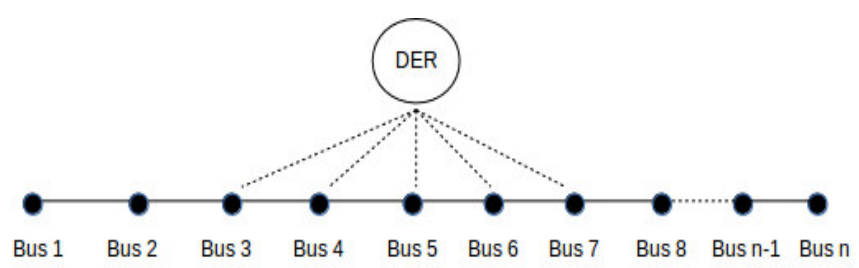

Fig. 1: Connection of the renewable source to the nearest points of the network

Another issue considered in this problem is the minimization of the equipment cost. However, in this type of problems where the functions in optimization are in conflict, normally they have a set of solutions and not a unique solution. To determine the optimal solutions set a multi objective approach is used. Because of its proven applicability in this type of problems, the evolutionary genetic algorithm NSGA-II (Fast Non-dominated Sorting Genetic Algorithm) was used. With this algorithm a trade-off between the several objective functions is obtained, thus obtaining the number and location of switching devices as well as the point of connection of the DER to the distribution network. The analysis of the problem considers the restriction of no-island operation. To improve the availability of the renewable sources and privilege the ones with higher generation power, under the improvement of network reliability, a composite index was introduced that privileges the DER with higher power. This composite index is based on the SAIDI index modified to consider also the DER.

\section{A. Pareto Optimal Front}

The resolution of this multi objective problem does not result in one solution but on a set of solutions, corresponding to a trade-off between the two objective functions. To obtain the optimal set of solutions a Pareto dominance approach was considered [24]. In general a multi objective optimization problem can be formulated considering that for a space $\mathrm{S}$ with $\mathrm{n}$-dimensional the determination of a vector $\mathrm{X}$ of decision variables that satisfies a given set of criteria depending on (1) will be required.

$$
\begin{aligned}
& \min \quad f(X)=\left(f_{1}(X), f_{2}(X), \ldots, f_{m}(X)\right) \\
& \text { s.t.: } x \in \Omega \\
& X=\left(x_{1}, x_{2}, \Lambda, x_{n}\right)
\end{aligned}
$$

Where $f_{1}(X), f_{2}(X), \ldots, f_{m}(X)$ are the $m$ objective functions that are conflicting with each other; $x_{1}, x_{2}, \lambda, x_{n}$ are the $\mathrm{n}$ optimization parameters, and $\Omega \in R^{n}$ is the solution or parameter space. The set of optimal solutions (that may be 
subjected to restrictions) consists of a set of solutions satisfying the objective functions in an acceptable level that are not dominated by another solution. When this occurs, we are in presence of a Pareto optimal set of solutions and any solution of this set cannot be improved without worsening at least on other objective function. The obtainment of a set of solutions permits the distribution operator to choose the best solution based on his criteria.

\section{B. Decision Variables}

In this multi objective optimization problem the aim is to find the number and location of switching devices as well as the point of connection of the DER to the distribution network. The optimization problem is treated as a true minimization problem with each function being minimized individually. The modulation of a radial network can be done as a tree with $i$ buses. In each bus it may be connected a line and/or a renewable source and/or a consumer. The switching devices are installed in the beginning of a line. By the definition of the problem the decisions variables are the point of connection of the DER, the number of switching devices and its location in the network. For the switching devices it will be considered a variable $\lambda$ according to (2). For the connection of a renewable source is considered a variable $\gamma$ as presented by (3).

$\lambda_{i}= \begin{cases}1, & \text { if a switching device is connected to bus } i \\ 0, & \text { otherwise }\end{cases}$

$Y_{i}=\left\{\begin{array}{lc}1, & \text { if a renewable generator is connected to bus } i \\ 0, & \text { otherwise }\end{array}\right.$

\section{Objective functions}

As described before the multi objective functions considered in this problem are: 1) the optimal location of DER connection to the network and the optimal location and number of switching devices and 2) the cost of equipment's. The above are designated by: 1) composite index $C I_{S A I D I-D G}$. and 2) $E C$ investment in equipment's and connections of DER.

\section{1) Objective Function: Location of DER and Number and} Location of Switching Devices

The location and number of switching devices is normally formulated taking into consideration the index SAIDI defined by the IEEE standard 1366 [25]. However, this standard does not consider the distribution networks with the integration of DER. In order to consider that integration a different index will be considered. This index has to privilege the availability of the most important renewable sources (with higher power of generated capacity). Thus, an index that integrates the classical SAIDI and the referred DER availability is used. This results in a composite index designated by $C I_{S A I D I-D G}$. The DER is integrated in this index considering that each generator is equivalent to a certain number of consumers in accordance to equation (4). Thus, the algorithm used to optimize the objective functions will try to maintain as much as possible the most important DER connected to the network.

$$
N_{D G m}=\frac{P_{D G m}}{P_{\text {AVcons }}}
$$

Where $N_{D G m}$ is the number of consumers representing the distributed generator $m\left(D_{G m}\right), P_{D G m}$ is the power $(\mathrm{kVA})$ of $D_{G m}$, and $P_{A V \text { cons }}$ is the average client power $(\mathrm{kVA})$ of the network. The mathematical model of the objective function $C I_{S A I D I-D G}$ considering the decision variables previously referred is defined by equation (5).

$$
\min \frac{\sum_{i}\left(\left(\lambda_{i} \times t_{S i} \times\left(N_{i}+N_{D G m i}\right)\right)+\left(\bar{\lambda}_{i} \times t_{N i} \times\left(N_{i}+N_{D G m i}\right)\right)\right)}{\left(N_{t}+\sum_{m} N_{D G m}\right)}
$$

Where $t_{S i}$ is the average outage time (minutes/year) of section $i$ with a switch affecting section $i ; t_{N i}$ is the average outage time (minutes/year) of section $i$ with no interruption device affecting section $i ; N_{I}$ is the number of clients in section $i ; N_{t}$ is the total number of clients in the network; $N_{D G m i}$ is the number of consumers representing generator $m$ according to eq. (4) and connected to section $i$.

\section{2) Objective Function:Equipment Cost}

The second objective function considered is the investment representing the cost of the equipment's and their installation. This objective function will be formulated in accordance with equation (6).

$$
E C=\sum_{i}\left(\left(\lambda_{i} \times C_{S i}\right)+\left(\gamma_{i} \times C_{D G m i}\right)+\left(\bar{\lambda}_{i} \times C_{N i}\right)\right)
$$

Where $C_{S i}$ is the cost of the switching device installed in section $i, C_{D G m i}$ is the cost of the connection of $D G_{m}$ to section $i$, and $C_{N i}(=0)$ for sections with no equipment.

\section{MultiobJective NSGA-II AlgORITHM}

For the minimization of the objective functions, the NSGAII algorithm was used. This algorithm is an elitist multi objective evolutionary algorithm characterized by a fast nondominated sorting and by an efficient crowding-distance assignment approach. This is a proven algorithm used in many applications with performance tested in many comparative studies [26], [27], [28], [29], [30]. Problems like sensitiveness to the shape of Pareto optimal front and dependence from the 
efficiency of the single objective optimizer that classical algorithms show are overpass by evolutionary algorithms. The set of solutions are subjected to a selection process, based on genetic operators that normally consist of recombination and mutation [28]. An elite-preserving strategy and an explicit diversity preserving mechanism are used by the NSGA-II algorithm. This is a mechanism that ensures and preserves diversity and guarantees a good convergence towards the Pareto front. The genetic algorithm NSGA-II has the following general solving procedure:

1. The initial population $N$ is randomly initiated, with each solution called a chromosome;

2. A new set of solutions are generated from the existing ones, using two genetic operators: crossover and mutation. The operator crossover selects two chromosomes (parents) based on their fitness value and combines them to form two new chromosomes (offspring). The genetic operator mutation introduces random changes at gene level to the chromosomes with a probability typically less than $1 \%$; with the crossover operator convergence is assured and with the mutation operator diversity is introduced to the population.

3. Each solution of the $2 \mathrm{~N}$ population is evaluated and a fitness value is assigned to each of them;

4. Based on the fitness value, $\mathrm{N}$ solutions are selected ;

5. The previous four steps are repeated until the stopping criterion is met.

The encoding of the problem in the genetic algorithm uses a matrix with $N$ lines (population) and $M$ columns for the location of the switching devices in the network. The buses are numerated in a sequential form, being each column directly related with the beginning of a bus. The existence of a " 1 " in a column means that there is a switching device in the bus. A " 0 " means the contrary.

For the encoding of the connections of the DER another matrix was used with $N$ lines (one line for each DER connected to the network) and $M$ columns (number of the bus connecting a DER to the network). In this case, each element is related with each generator. Inside of each element there will be placed a sequential number from 1 to $\mathrm{N}$ (where $\mathrm{N}$ is the number of possible connection points). The number 1 corresponds to the nearest point, the 2 the second nearest point and so on.

\section{RESUlts AND ANALYSES}

A Portuguese distribution network was used to test the effectiveness of the proposed approach. In Figure 1 is presented this distribution network, with $15 \mathrm{kV}$ network and 94 buses. Four renewable sources were considered with $350 \mathrm{~kW}$ (G1), $1150 \mathrm{~kW}$ (G2), $550 \mathrm{~kW}$ (G3) and $800 \mathrm{~kW}$ (G4) respectively. For each generator it was considered three possible connections to the network (Fig. 1 in different colors dots for each DER).

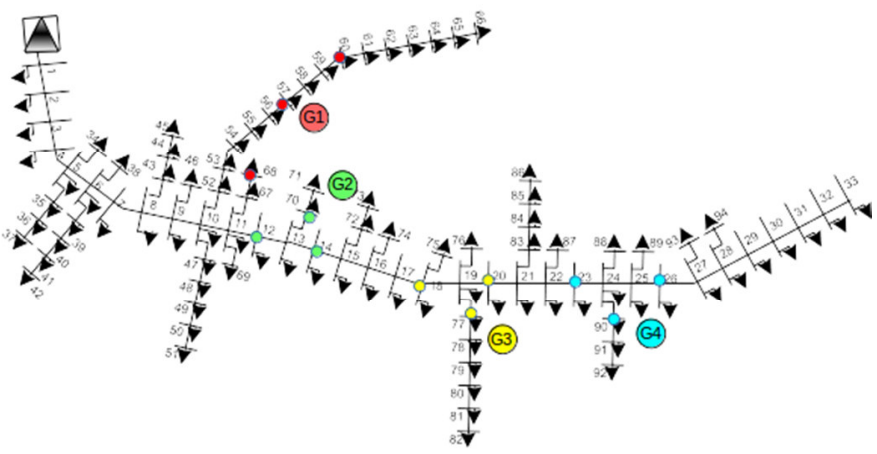

Fig. 2: Configuration of the 94 Bus Portuguese $15 \mathrm{kV}$ MV Distribution Network Test Case.

Through the implementation of the algorithm it was possible to obtain the best Pareto-front set of results considering the objective functions Composite Index and Equipment Cost as presented in Figure 2. From this figure it is possible to verify that the obtained results form a Pareto front. It is also possible to verify that the Pareto front is generally well defined and the solutions are well spread.

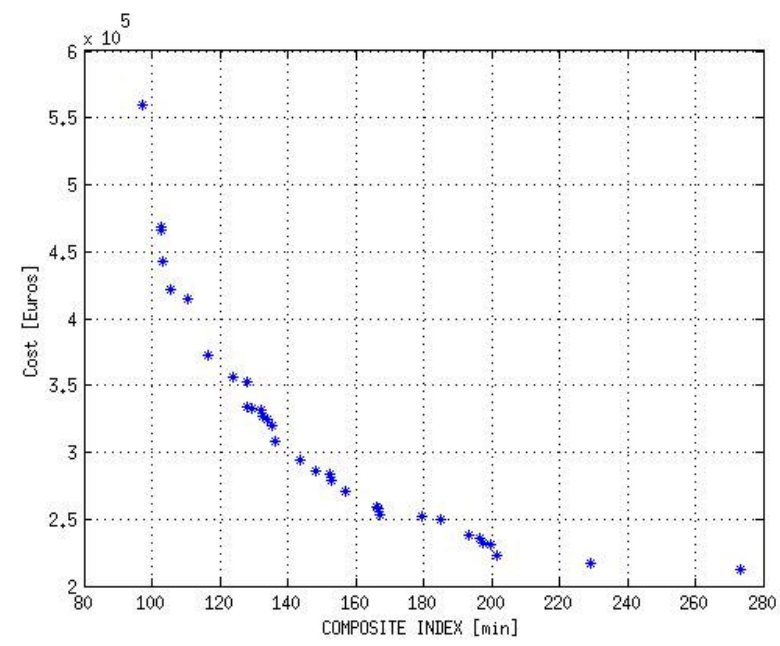

Fig. 3 - Non-dominated solutions obtained from de NSGAII algorithm considering as objective functions - Composite Index and Equipment Cost.

In Table I is presented the numerical values of the best solutions for each of the objective functions (extreme solutions of the Pareto front). From these results is possible to verify the conflict between those objective functions. In fact, for the solution with lower cost it is obtained the maximum composite index, and vice-versa.

TABLE I. NUMERICAL RESULTS OF THE BEST SOLUTIONS FOR EACH OF THE OBJECTIVE FUNCTIONS

\begin{tabular}{|c|c|}
\hline $\begin{array}{c}\text { Composite } \\
\text { Index (min) }\end{array}$ & $\begin{array}{c}\text { Equipemnt Cost } \\
\text { (Euros) }\end{array}$ \\
\hline 273,2537 & 212500 \\
97,0915 & 559500 \\
\hline
\end{tabular}


In table II is presented the number and location of the switching devices and the location of the bus where the generators will be connected. It must be noted that for the possible connections are for first generator 1 - buses 60,57 and 68, for generator $2-$ buses 70,12 and 14, for the third generator - buses 77,18 and 20 and for generator 4 - buses 90,23 and 26.

TABLE II. LOCATION IN THE NETWORK OF THE INTERRUPTION DEVICES OBTAINED FOR THE BEST SOLUTIONS FOR EACH OBJECTIVE FUNCTION

\begin{tabular}{|c|c|}
\hline Objective Function & Interruption Devices Placement \\
\hline \multirow[t]{2}{*}{ Min Equipment Cost } & 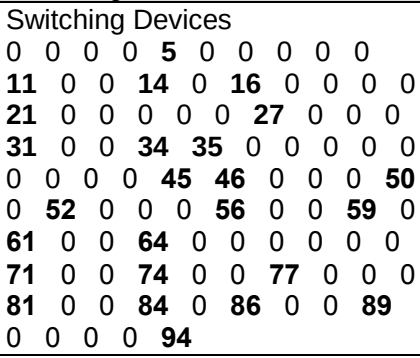 \\
\hline & $\begin{array}{l}\text { Distribution Energy Resources } \\
\mathbf{5 7 , 7 0 , 7 7 , 9 0}\end{array}$ \\
\hline \multirow[t]{2}{*}{ Min Composite Index } & 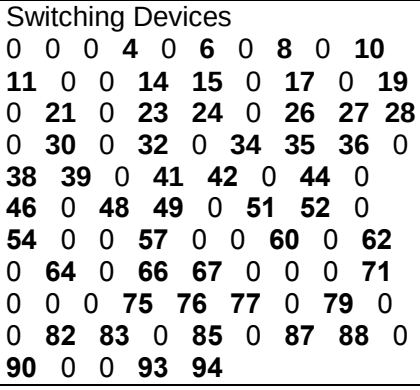 \\
\hline & $\begin{array}{l}\text { Distribution Energy Resources } \\
\mathbf{6 8}, \mathbf{1 4}, \mathbf{2 0}, \mathbf{2 6}\end{array}$ \\
\hline
\end{tabular}

The final set of results is the output of the NSGA-II algorithm in the form of a Pareto front. Each solution of the Pareto front represents a specific solution that is optimal in its own way considering the two objective functions.

TABLE III. IMPROVEMENT OF DISTRIBUTION NETWORK RELIABILITY AND DISTRIBUTED ENERGY RESOURCES AVAILABILITY FOR THE BEST INDIVIDUAL SOLUTIONS.

\begin{tabular}{|c|c|c|}
\hline Solution & SAIDI (min) & $\begin{array}{c}\text { DER } \\
\text { Unavailability } \\
\text { (min) }\end{array}$ \\
\hline $\begin{array}{c}\text { Best interms of } \\
\text { reliability }\end{array}$ & 92,5621 & 69,1107 \\
\hline $\begin{array}{c}\text { Best in terms } \\
\text { of cost }\end{array}$ & 268,4377 & 233,8619 \\
\hline
\end{tabular}

In Table III we can see the improvements of distribution network reliability and Distributed Energy Resources availability for the best individual solutions. Relatively to the initial distribution network reliability $(351 \mathrm{~min})$ we can see that for the best reliability solution there was a reduction of the SAIDI index in $73 \%$ and for the DER unavailability a reduction of $80 \%$. For the best cost solution there was reduction of the SAIDI index in $24 \%$ and for the DER unavailability a reduction of $33 \%$. The results show that when the Equipment Cost increases the SAIDI Index decreases and vice-versa. From the results of the Pareto front it is possible for the decision maker of the distribution operator, to select a particular solution based on a defined criteria for one objective function assuring that the other objective function has the best possible value.

\section{CONCLUSIONS}

This work proposes a multi objective optimization of the location of DER connection considering the switching devices placement to increase network reliability and availability of DER, reducing at the same time the equipment cost in a no island operation environment. Thus, through the optimal connection of DER to the distribution network and the allocation of the switching devices is achieved an improvement of distribution network reliability and Distributed Energy Resources availability. The objective functions considered for the minimization problem were two: 1) location of DER and number and location of Switching Devices and 2) the cost of the equipment's. For the first objective function it was considered a composite index that is a result of the introduction of the DER in the SAIDI index. This considered the no island operation of the distribution network. To resolve this minimization problem the NSGA-II genetic algorithm was used because of its proven applicability to this type of problems. To obtain the non-dominated solutions a Pareto optimal front concept was considered. This algorithm was applied to a real distribution $15 \mathrm{kV}$ medium voltage network with 94 buses and four renewable sources with different capacities. The obtained results show a reduction of the SAIDI index and DER unavailability relatively to the initial network reliability and distribution network unavailability. From the Pareto front solutions, it is possible to choose a solution based on specific criteria resulting in a useful tool for the planning of a distribution operator.

\section{ACKNOWLEDGMENT}

This work was supported by national funds through FCT Fundação para a Ciência e a Tecnologia, under projects UID/CEC/50021/2013 and UID/EEA/00066/2013.

\section{REFERENCES}

[1] H. Brown, S. Suryanarayanan and G. T. Heydt, "Some characteristics of emerging distribution systems considering the smart grid initiative," The Electricity Journal, Vols. 23, Issue 5, 2010.

[2] H. Farhangi, "The path to the smart grid",IEEE Power and Energy Magazine, 2010.

[3] V. F. Martins, C. Borges, "Active distribution network integrated planning incorporating distributed generation and load response uncertainties", IEEE Transactions on Power Systems, Vol. 26, pp. 21642172, 2011

[4] W. Sheng, K. Liu, X. Meng, X. Ye and Y. Liu, "Research and practice on typical modes and optimal allocation method for PV-Wind-ES in microgrid," Electric Power Systems Research, 2014.

[5] M. F. Akorede, H. Hizam, E. Pouresmaeil, "Distributed energy resources and benefits to the environment", Renewable and Sustainable Energy Reviews, Vol.14, 724-734, 2010.

[6] G. Balakrishna and C. S. Babu, "Optimal placement of switches in DG equipped distribution systems by particle swarm optimization," 
International Journal of Advanced Research in Electrical, Electronics and Instrumentation Engineering, Vols. 2, Issue 12, 2013.

[7] S. Gangury, N. C. Sahoo and D. Das, "Multiobjective particle swarm optimization based on fuzzy-Pareto-dominance for possibilistic planning of electrical distribution systems incorporating distributed generation," Fuzzy Sets and Systems, vol. 213, pp. 47-73, 2013.

[8] S. Golestani and M. Tadayon, "Optimal Switch placement in distribution power system using linear fragmented particle swarm optimization algorithm preprocessed by GA," 8th International Conference on the European Energy Market, 2011.

[9] S. H. Mousavi and M. Mohammadi, "Optimal planning of distribution system in presence of DG with considering economic and reliability indices with fuzzy logic algorithm," Australian Journal of Basic and Applied Sciences, vol. 5(8), pp. 1248-1256, 2011.

[10] Z. N. Popovic, V. D. Kerleta and D. S. Popovic, "Hybrid simulated annealing and mixed integer linear programming algorithm for optimal planning of radial distribution networks with distributed generation," Electric Power Systems Research, vol. 108, pp. 211-222, 2014.

[11] Z. Li and X. Z. ,. W. Yuqin, "Research on optimization of recloser placement of DG enhanced distrib40ution networks," DRPT, 6-9 April, Nanjing, , 2008.

[12] E. Jalilzadeh, S. M. Miri-Larimi and M. R. Haghifam, "Optimal placement of sectionalizing switches in distribution network with presence of renewable energy resources," CIRED Workshop, Lisbon, 2012.

[13] V. Calderano, V. Lattarulo, A. Piccolo and P. Siano, "Optimal switch placement by alliance algorithm improving microgrids reliability," IEEE Transactions on Industrial Informatics, Vols. 8, $\mathrm{n}^{\circ}$ 4, 2012.

[14] S. A. M. Javadian and M. R. Haghifam, "Optimal placement of protective devices in distribution networks based on risk analysis," Transmission and Distribution Conference and Exposition, Latin America, IEEE/PES , 2010.

[15] A. V. Pombo, J. M. Pina, V. F. Pires, "Application of NSGA-II algorithm to multiobjective optimization of switching devices placement in electric distribution systems", 5th Doctoral Conference on Computing, Electrical and Industrial Systemsk, DoCEIS 2014, Costa Caparica, Portugal, 380-387, 2014.

[16] A. V. Pombo, J. M. Pina, V. F. Pires, "Multiobjective planning of distribution networks incorporating switches and protective devices using a memetic optimization", Reliability Engineering \& System Safety, Vol. 136, pp. 101-108, April 2015

[17] A. Alarcon-Rodriguez, G. Ault, S. Galloway, "Multi-objective planning of distributed energy resources: A review of the state-of-the-art", Renewable and Sustainable Energy Reviews, Vol. 14,pp. 1353-1366, 2010.

[18] P. S. Geogilakis and N. D. Hatziargyriou, "Optimal distribution generation placement in power distribution networks: models, methods and future research," IEEE Transactions on Power Systems, Vols. 28, $\mathrm{N}^{\mathrm{o}}$ 3, 2013.

[19] S. Sohrabijam and S. Mohammadi, "Optimal Placement of Distribution Generation Resources in Distribution Systems via Reliability Criterion," Computing, Information Systems \& Development Informatics, Vols. 3, No 4, 2012.

[20] C. L. T. Borges and D. M. Falcão, "Optimal distributed generation allocation for reliability, losses, and voltage improvement," Electrical Power and Energy Systems, vol. 28, pp. 413-420, 2006.

[21] N. Khalesi, N. Rezai and M. R. Haghifam, "DG allocation with application of dynamic programming for loss reduction and reliability improvement," Electrical Power and Energy Systems, vol. 33, pp. 288295, 2011.

[22] G. Celli, S. Mocci, F. Pilo, G. G. Soma, “A multi-objective approach for the optimal distributed generation allocation with environmental constraints", Proceedings of the 10th International Conference on Probabilistic Methods Applied to Power Systems, 2008.

[23] V. Pombo, J. Murta-Pina, V. F. Pires, “A Multiobjective Placement of Switching Devices in Distribution Networks Incorporating Distributed Energy Resources," Electric Power Systems Research, vol. 130, pp. 3445, January 2016.
[24] W. Tan, M. Y. Hassan, M. S. Majid, H. A. Rahman, "Optimal distributed renewable generation planning: A review of different approaches", Renewable and Sustainable Energy Reviews, Vol. 18, 626645, 2013.

[25] IEEE Power Engineering Society. (May 14). IEEE Std. 1366:-2003 IEEE guide for electric power distribution reliability indices. New York: Institute of Electrical and Electronics Engineers, Inc; 2004; 35 (ISBN 07381-3890-8 SS95193).

[26] A. Zidan, M. F. Shaaban and E.-S. E. F, "Long-term multiobjective distribution network planning by DG allocation and feeder's reconfiguration," Electric Power Systems Research, vol. 105, pp. 95104, December 2013.

[27] C. H. Barbosa, R. F. Alexandre and J. A. Vasconcelos, "A practical codification and it'sanalysis for the generalized reconfiguration problem," Electric Power Systems Research, vol. 97, pp. 19-33, April 2013.

[28] W. Sheng, K. Liu, X. Meng, X. Ye and Y. Liu, "Research and practice on typical modes and optimal allocation method for PV-Wind-ES in microgrid," Electric Power Systems Research, vol. 120, pp. 242-255, March 2015.

[29] M. T. Al-Hajri, M. A. Abido, "Assessment of Genetic Algorithm selection, crossover and mutation techniques in reactive power optimization”, IEEE Congress on Evolutionary Computation - CEC, pp. 1005-1011, 2009.

[30] K. Deb, "Multi-objective optimization using evolutionary algorithms," Wiley, 9506, 2001. 